\title{
Wave Instabilities in Excitable Media with Fast Inhibitor Diffusion
}

\author{
V.S. Zykov, ${ }^{1}$ A. S. Mikhailov, ${ }^{2}$ and S.C. Müller ${ }^{1}$ \\ ${ }^{1}$ Institut für Experimentelle Physik, Otto-von-Guericke-Universität, Universitätsplatz, 2, D-39106 Magdeburg, Germany \\ ${ }^{2}$ Fritz-Haber-Institut der Max-Planck-Gesellschaft, Faradayweg 4-6, D-14195 Berlin, Germany
}

(Received 10 April 1998)

\begin{abstract}
An excitable activator-inhibitor system with relatively fast inhibitor diffusion is considered. Numerical simulations of wave propagation inside long channels show transitions from stable flat traveling waves to folded waves and further to spreading spiral turbulence as the inhibitor diffusivity is increased. For sufficiently narrow channels the suppression of turbulence and the development of regular steadily propagating patterns is observed. The curvature dependence of the wave propagation velocity is derived and used to interpret the observed phenomena. [S0031-9007(98)07163-4]
\end{abstract}

PACS numbers: 82.20.Wt, 05.70.Ln, 82.40.Ck

Excitable media represent a wide class of nonequilibrium activator-inhibitor systems that play an important role in physical, chemical, and biological applications [1-3]. They show a rich potential for spatiotemporal pattern formation and can support propagation of single or periodic traveling excitation waves, as well as more complex regimes, such as rotating spiral waves and turbulence. Until recently, the principal attention in studies of wave instabilities in such media has been paid to the systems with nondiffusing or weakly diffusing inhibitor species $[4,5]$. The instabilities of propagating excitation waves in media with relatively fast inhibitor diffusion are less investigated (see, however, [6]). The transverse front instabilities due to fast diffusion of an inhibitor species have been previously discussed for bistable activator-inhibitor models, e.g., in the context of the combustion theory $[7,8]$ and propagating chemical fronts separating two steady states [9]. Such instabilities can lead to the development of complex labyrinthine patterns and spiral wave turbulence [10]. An important role in the stability analysis of propagating fronts in the bistable media is played by the curvature dependence of their velocity $[7,8,10,11]$.

In this Letter we investigate transverse instabilities of traveling excitation waves caused by fast inhibitor diffusion. The curvature dependence of the propagation velocity for such waves is derived and its properties are used to explain the observed transitions from a stable flat wave to a folded one and then to spiral turbulence.

We consider wave propagation in two-dimensional excitable media using a general two-component reactiondiffusion system (cf. [12,13])

$$
\begin{aligned}
& \frac{\partial u}{\partial t}=\nabla^{2} u+F(u, v), \\
& \frac{\partial v}{\partial t}=\sigma \nabla^{2} v+\epsilon G(u, v),
\end{aligned}
$$

for the activator $u=u(x, y, t)$ and inhibitor $v=$ $v(x, y, t)$. Here the activator diffusion constant is chosen to be unity by appropriate scaling of the space coordinates. We study the situation when $\sigma>1$, i.e., inhibitor is diffusing faster than the activator. Our numerical analysis is performed for a frequently used Rinzel-Keller-type model with the following nonlinear functions (see [13]):

$$
\begin{aligned}
F(u, v) & =f(u)-v, \\
f(u) & =-k_{1} u, \quad u<\delta, \\
& =k_{f}(u-a), \quad \delta<u<1-\delta, \\
& =k_{2}(1-u), \quad 1-\delta<u, \\
G(u, v) & =k_{g} u-v,
\end{aligned}
$$

with $k_{f}=2, k_{g}=2, a=0.1, \delta=0.01$. The parameters $k_{1}$ and $k_{2}$ are chosen in such a way that the function $f(u)$ is continuous at $u=\delta$ and $u=1-\delta$.

The model has a single steady state which is stable with respect to small perturbations. Application of a sufficiently strong local perturbation gives rise to a stable traveling pulse in a one-dimensional medium or to an expanding circular wave in a two-dimensional medium. The latter eventually approaches a steady flat wave at large radius propagating at the velocity

$$
V_{p}=V_{p}(\epsilon, \sigma)
$$

that can be computed or determined analytically $[10,12,13]$. Steady propagating pulses and flat waves are possible however only in a certain portion of the $\sigma-\epsilon$ parameter plane. Their existence boundary $\epsilon=\epsilon_{c}(\sigma)$ has been computed by us for the model (1) and (2); it is shown by the solid curve $\Gamma_{1}$ in Fig. 1 . No steady propagating waves with flat fronts exist above this boundary, although the curved waves may still propagate there. To study an example of the evolution of locally induced waves systematically in the vicinity of such a boundary we integrated Eqs. (1) and (2) numerically for different values of $\epsilon$ and $\sigma$. In particular we chose the parameters along the straight line $\epsilon / \sigma=0.075$ crossing the existence boundary of flat waves at $\epsilon=0.15$ and $\sigma=2.0$.

The computations were carried out with the constant time step $\Delta t=0.005$ and the space step $\Delta x=0.2$. The integrated medium represented a long channel with noflux boundaries for both species. As the initial condition, 


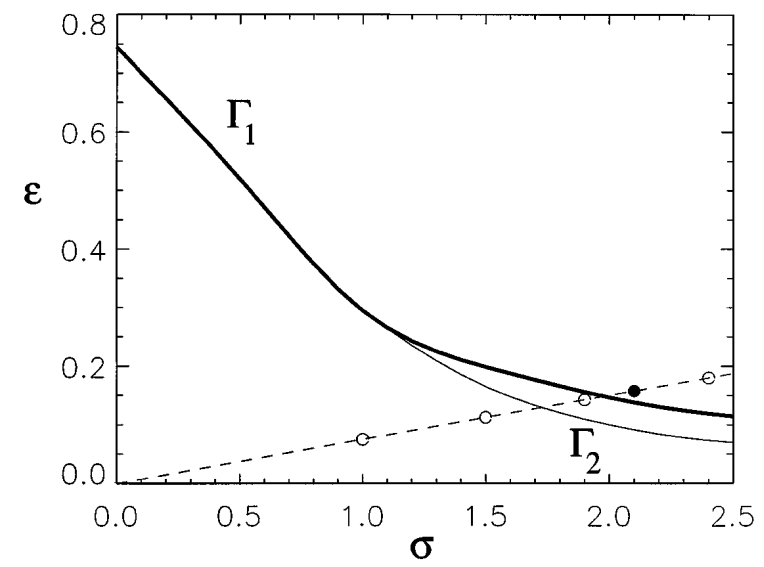

FIG. 1. The boundaries of steady propagation $\Gamma_{1}$ and transverse instability $\Gamma_{2}$ of flat waves. The circles show the parameter values that are used to compute the curvature dependence of the propagation velocity.

we used the uniform steady state of this system and then stimulated the two right corners to create waves propagating to the left. After collision of the two expanding waves, a single wave with a flat front was quickly established if the parameters were chosen from the region below the existence boundary and relatively far from it (e.g., for the points on the straight line in Fig. 1 with $\sigma=1.0$ or $\sigma=1.5$ ). However, for the point with $\sigma=$ 1.9 the same initial condition resulted in the development of a traveling folded wave with characteristic ripples [Fig. 2(a)]. We did not find that this folded wave eventually breaks up, even when the propagation was followed by us over a long distance using the comoving integration frame. The wave shape was not stationary and the ripples randomly wandered across the channel. It was shown that initial stimulation of a whole right edge leads asymptotically to the same regime.

In contrast to this, breakups of waves have always been observed in our simulations in the parameter region above the existence boundary of flat waves (e.g., for the points with $\sigma=2.1$ or $\sigma=2.4$ in Fig. 1). Note that in this parameter region a flat wave induced at the right edge
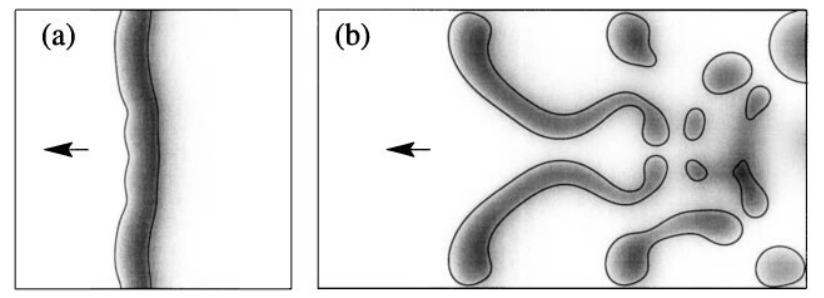

FIG. 2. Waves propagating down the channel with excitable medium for (a) $\epsilon=0.1425, \sigma=1.9$ and (b) $\epsilon=0.1575, \sigma=$ 2.1. The gray-scale images of the inhibitor field $v$ and the contours of the activator field with $u=0.5$ are shown at time instants (a) $t=1500$ and (b) $t=400$ in the comoving frame. The frame sizes are (a) $120 \times 120$ and (b) $160 \times 90$. disappeared with time. The two waves expanding from the corners did not merge to produce a single wave. The heads of the two waves moved at a constant velocity and their shapes remained practically fixed. However, the form and the size of their tails varied with time. They elongated and broke up, creating new islands of excitation that started to expand and to interact with each other. As a result of a series of breakups and collisions a very irregular wave pattern of spiral turbulence developed behind the leading waves [Fig. 2(b)].

To explain spatiotemporal regimes found in numerical simulations we analyze the curvature dependence of the propagation velocity $V$ for the general system (1). If the wave is thin as compared with the radius of curvature, its front and rear sides have the same curvature $K$. Under this condition the solution of Eqs. (1) for the wave propagating steadily with a stationary profile satisfies the equations (cf. $[12,13])$

$$
\begin{gathered}
(V+K) \frac{d u}{d \xi}=\frac{d^{2} u}{d \xi^{2}}+F(u, v), \\
(V+\sigma K) \frac{d v}{d \xi}=\sigma \frac{d^{2} v}{d \xi^{2}}+\epsilon G(u, v),
\end{gathered}
$$

where $\xi=r+V t$ is the coordinate in the coordinate system moving with the wave.

System (4) can be reduced to a similar system with a zero curvature $K$, following $[14,15]$, by multiplying the second equation of the system (4) with

$$
\gamma=\frac{V+K}{V+\sigma K}
$$

With the notations $V^{*}=V+K, \epsilon^{*}=\gamma \epsilon, \sigma^{*}=\gamma \sigma$ and we write Eqs. (4) in the form

$$
\begin{aligned}
& V^{*} \frac{d u}{d \xi}=\frac{d^{2} u}{d \xi^{2}}+F(u, v), \\
& V^{*} \frac{d v}{d \xi}=\sigma^{*} \frac{d^{2} v}{d \xi^{2}}+\epsilon^{*} G(u, v) .
\end{aligned}
$$

We note next that Eqs. (6) coincide with Eqs. (4) describing steady propagation of a flat wave $(K=0)$ in system (1) with the renormalized parameters $\epsilon^{*}$ and $\sigma^{*}$. This implies that $V^{*}=V_{p}\left(\epsilon^{*}, \sigma^{*}\right)$. Therefore we have

$$
V=V_{p}(\gamma \epsilon, \gamma \sigma)-K
$$

where $\gamma$ is given by (5).

It is convenient to introduce the function $V_{\epsilon, \sigma}(\gamma) \equiv$ $V_{p}(\epsilon \gamma, \sigma \gamma)$, treating $\gamma$ as an independent argument. Note that varying $\gamma$ is equivalent to moving along a ray $\epsilon / \sigma=$ const in the parameter plane $\sigma-\epsilon$. As an example, we have computed this function (accuracy about $\pm 1 \%$ ) by integrating Eqs. (1) for the point $\epsilon=0.1575$ and $\sigma=2.1$ (the filled circle in Fig. 1) laying on the straight line $\epsilon / \sigma=0.075$ in the parameter plane $\sigma-\epsilon$. Note that since the filled circle in Fig. 1 is located above the existence boundary of flat waves, the system in this case does not support propagation of flat waves at $\gamma=1$. The flat wave solutions are possible only for $\gamma<\gamma_{c}<1$, where $\gamma_{c}=0.92$ as shown in Fig. 3. 
Taking into account that $V+K=V_{\epsilon, \sigma}(\gamma)$ and using Eqs. (5) and (7), we find that the velocity $V$ of a traveling wave with curvature $K$ is determined by two algebraic equations:

$$
\begin{gathered}
V_{\epsilon, \sigma}(\gamma)=K \frac{\gamma(\sigma-1)}{1-\gamma}, \\
V=V_{\epsilon, \sigma}(\gamma)-K .
\end{gathered}
$$

The solutions of Eq. (8) can be analyzed by simultaneously plotting the function $V_{\epsilon, \sigma}(\gamma)$ vs $\gamma$ and the hyperbola, representing the right-hand side of this equation (see, e.g., Fig. 3). The intersection of the two graphs is the root of Eq. (8). As the curvature $K$ is decreased, the hyperbola shifts down and to the right in this plot, until it ceases eventually to intersect the graph of the function $V_{\epsilon, \sigma}(\gamma)$ and the solution disappears. Hence, the solution exists in this case only if $K>K_{c}>0$, where $K_{c}$ is the critical curvature at which the hyperbola touches the graph of the function $V_{\epsilon, \sigma}(\gamma)$ at its end point (this last hyperbola is shown as the dotted curve in Fig. 3). Thus, we see that if the excitable medium does not support propagation of flat waves, traveling convex waves $\left(K>K_{c}>0\right)$ still exist in this system. Using similar arguments, it can be shown that, when propagation of flat waves is possible and $\sigma>1$, concave waves $(K<0)$ can propagate only if their curvature is sufficiently small.

Figure 4 shows the dependences of the propagation velocity on curvature for different values of the parameters $\sigma$ and $\epsilon$ taken along the dashed line in Fig. 1 as obtained by numerically solving Eqs. (8) and (9). When the diffusion constants of the activator and inhibitor species are equal, i.e., $\sigma=1$, the plot of $V(K)$ is a straight line with the slope $d V / d K=-1$ (cf. [16]). When the value of parameter $\sigma$ is increased, the solutions for the propagating concave waves are possible only if their curvature is not too strong (the curves for $\sigma=1.5$ and $\sigma=1.9$ in Fig. 4). Increasing further the ratio $\sigma$ of the two diffu-

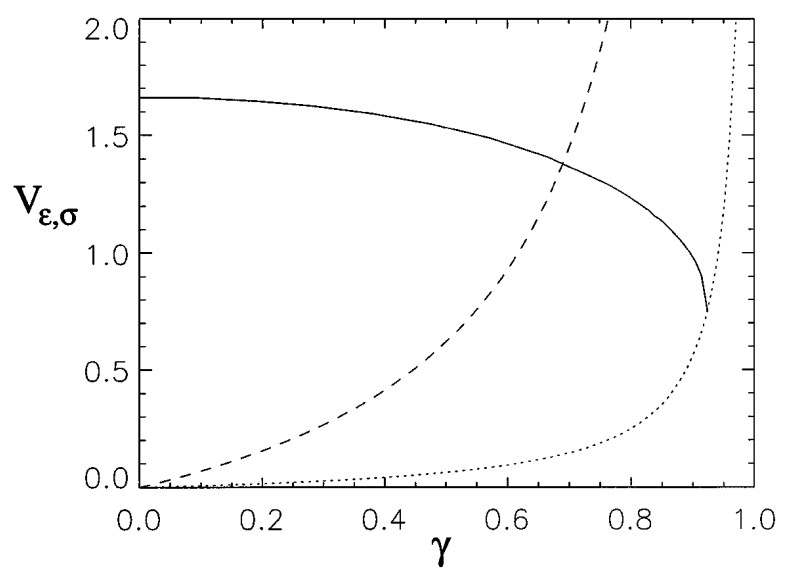

FIG. 3. The propagation velocity $V_{\epsilon, \sigma}(\gamma)$ of a flat wave for $\epsilon=0.1575$ and $\sigma=2.1$ (solid curve). The dashed and dotted curves show the right-hand side of Eq. (8) plotted for $K=0.57$ and $K=K_{c}=0.057$, respectively. sion constants, we see that the propagation of flat waves is no longer possible, but convex waves still exist if their curvature is sufficiently strong (the curves for $\sigma=2.1$ and $\sigma=2.4$ in Fig. 4).

The graphs for the curvature dependence of the propagation velocity can be used to discuss the stability of propagating waves and explain the development of turbulent regimes. It is known that propagation of flat waves is stable only if the condition $d V / d K<0$ at $K=0$ is satisfied [8]. Examining Fig. 4, one notes that this slope becomes positive shortly before the solutions for the flat traveling waves disappear under increasing $\sigma$. The solid thin curve $\Gamma_{2}$ in Fig. 1 shows the boundary determined by the equation $d V / d K=0$ at $K=0$. In the narrow region between this boundary and the curve $\Gamma_{1}$ in Fig. 1, flat waves exist but they are unstable with respect to the transverse instability. The folded nonbreaking wave, seen in Fig. 2(a), corresponds to the parameters $\epsilon$ and $\sigma$ chosen inside this narrow region. On the other hand, the repetitive breakups of waves and spreading of turbulence in Fig. 2(b) have been found for the parameters $\epsilon$ and $\sigma$ lying above the existence boundary of flat waves in Fig. 1.

The velocity-curvature relation shown in Fig. 4 permits steady propagating convex waves even in the region above the boundary $\Gamma_{1}$. We have attempted to produce such propagating convex waves by gradually decreasing the width of the channel in our simulations. Figure 5 gives, in the comoving coordinate frame, subsequent snapshots of a propagating pattern observed in a narrow channel by integration of Eqs. (1) with the parameters corresponding to the filled circle in Fig. 1. The same parameter values and similar initial conditions as for the simulation of spreading spiral turbulence in the wider channel [Fig. 2(b)] were used here. However, the wave propagation pattern inside the narrow channel was different. Initially, spiral turbulence developed [Fig. 5(a)], but later a thin elongated band appeared in the frontal part

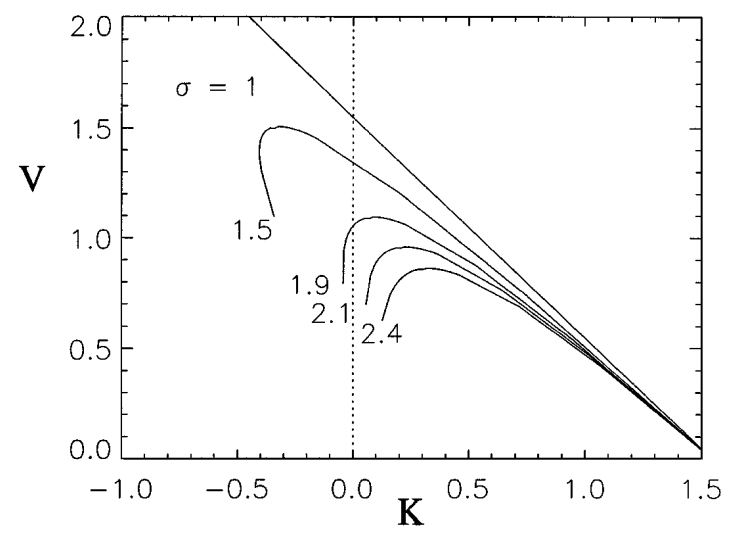

FIG. 4. The curvature dependence of the propagation velocity for different values of the parameters $\epsilon$ and $\sigma$ along the straight dashed line in Fig. 1. 

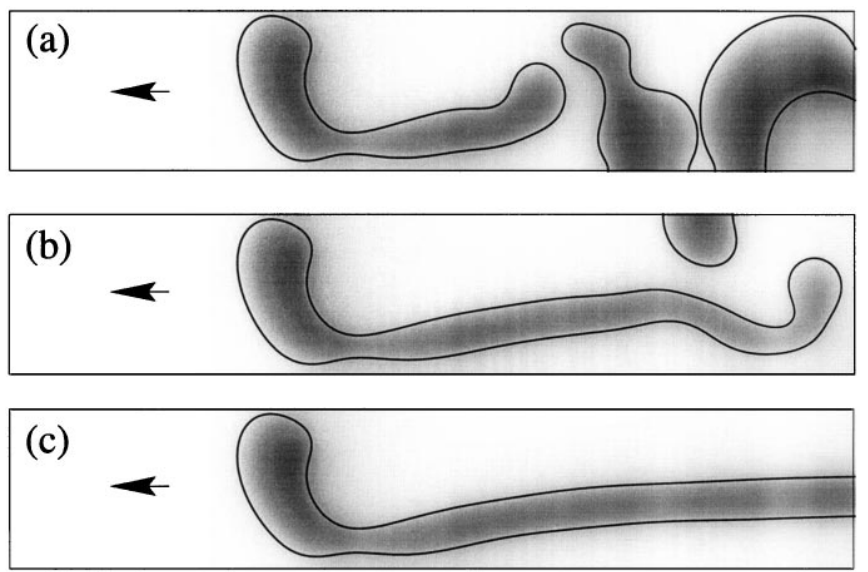

FIG. 5. Wave propagation in a narrow channel with $\epsilon=$ 0.1575 and $\sigma=2.1$. Gray scale and contour lines as in Fig. 2. The snapshots (a), (b), and (c) correspond, respectively, to the time instants $t=500,570$, and 800 . The frame size is $160 \times 30$.

of the spreading pattern [Fig. 5(b)]. The leading convex head of this band moved at a constant velocity and had a stationary shape. Its curvature was approximately equal to that of the fastest propagating curved wave permitted according to Fig. 4. The rear end of this band moved, however, more slowly and therefore the band continued to stretch along the channel, tending to occupy it completely after a long time [Fig. 5(c)].

In conclusion, two boundaries in the parameter plane $\sigma-\epsilon$ play an important role for propagating excitation waves in the considered system. The first of them $\left(\Gamma_{1}\right.$ in Fig. 1) limits the region, where steady propagation of flat waves is possible. The second boundary $\left(\Gamma_{2}\right.$ in Fig. 1$)$ is determined by the condition that $d V / d K=0$ at $K=0$. It corresponds to a bifurcation resulting in the transverse instability of a flat excitation wave. In the parameter region between these two boundaries the computations for the channels of various widths show the development of folded waves that do not undergo a spontaneous breakup. The spiral turbulence and repeated breakups of waves have been observed only above the first boundary $\Gamma_{1}$ in the $(\sigma-\epsilon)$ parameter plane. We found moreover that the spiral turbulence is suppressed in relatively narrow channels and complex regular regimes are realized instead which depend on the initial stimulation procedure and the width of the channel.

Stabilization of turbulent reaction fronts in narrow channels has been recently found for bistable chemical reactions [17]. However, the observed propagating structures were then different and represented folded fronts separating two steady states. The folded waves, seen in our computations [Fig. 2(b)], look similar to the wave patterns observed in the experiments with the BelousovZhabotinsky reaction $[18,19]$, but it is not yet clear whether these effects have the same theoretical explanation. It should be noted that our simulations in this Letter were performed for relatively low ratios of the inhibitor and activator diffusion constants $(\sigma \simeq 2)$ which enhances the possibility of a corresponding experimental observation in aqueous excitable medium.

[1] A.T. Winfree, When Time Breaks Down (Princeton University Press, Princeton, 1987).

[2] Spatio-Temporal Organization in Nonequilibrium Systems, edited by S.C. Müller and Th. Plesser (Projekt Verlag, Dortmund, 1992).

[3] Chemical Waves and Patterns, edited by R. Kapral and K. Showalter (Kluwer, Dordrecht, 1995).

[4] M. Bär, M. Hildebrand, M. Eiswirth, M. Falcke, H. Engel, and M. Neufeld, Chaos 4, 499 (1994).

[5] A. Karma, Chaos 4, 461 (1994).

[6] A. F. M. Maree and A. V. Panfilov, Phys. Rev. Lett. 78, 1819 (1997).

[7] G. H. Markstein, J. Aeronaut. Sci. 3, 18 (1951).

[8] Ya. B. Zeldovich, G. I. Barenblatt, V. B. Librovich, and G. M. Makhviladze, Mathematical Theory of Combustion and Explosion (Consultants Bureau, New York, 1985).

[9] K.J. Lee, W.D. McCormick, Q. Quang, and H.L. Swinney, Science 261, 192 (1993).

[10] A. Hagberg and E. Meron, Phys. Rev. Lett. 72, 2494 (1994).

[11] R. C. Brower, D. Kessler, J. Koplik, and H. Levine, Phys. Rev. A 29, 1335 (1984).

[12] A. S. Mikhailov, Foundation of Synergetics 1. Distributed Active Systems (Springer-Verlag, Berlin, 1990).

[13] V.S. Zykov, Simulation of Wave Processes in Excitable Media (Manchester University Press, Manchester, 1987).

[14] V.S. Zykov, Biophysics (USSR) 25, 906 (1980).

[15] A.S. Mikhailov and V.S. Zykov, Physica (Amsterdam) 52D, 379 (1991).

[16] J. J. Tyson and J. P. Keener, Physica (Amsterdam) 32D, 327 (1988).

[17] D. Horvath, V. Petrov, S. K. Scott, and K. Showalter, J. Chem. Phys. 98, 6332 (1993).

[18] Zs. Nagy-Ungvarai and S.C. Müller, Int. J. Bifurcation Chaos 4, 1257 (1994).

[19] M. Markus, G. Kloss, and I. Kusch, Nature (London) 371, 402 (1994). 